\title{
Clinical features and the traditional Chinese medicine therapeutic characteristics of 293 COVID-19 inpatient cases
}

\author{
Zixin Shu ${ }^{1, *}$, Yana Zhou ${ }^{2, *}$, Kai Chang ${ }^{1, *}$, Jifen Liu ${ }^{2}$, Xiaojun Min $^{2}$, Qing Zhang ${ }^{2}$ Jing Sun ${ }^{2}$, Yajuan Xiong ${ }^{2}$, \\ Qunsheng Zou ${ }^{1}$, Qiguang Zheng ${ }^{1}$, Jinghui Ji ${ }^{1}$, Josiah Poon $(\bowtie)^{4,5}$, Baoyan Liu $(\bowtie){ }^{6}$, Xuezhong Zhou $(\bowtie)^{1}$, \\ Xiaodong $\operatorname{Li}(\bowtie)^{2,3}$ \\ ${ }^{1}$ Institute of Medical Intelligence, School of Computer and Information Technology, Beijing Jiaotong University, Beijing 100044, China; \\ ${ }^{2}$ Hubei Provincial Hospital of Traditional Chinese Medicine, Wuhan 430061, China; ${ }^{3}$ Institute of Liver Diseases, Hubei Provincial Academy \\ of Traditional Chinese Medicine, Wuhan 430061, China; ${ }^{4}$ School of Computer Science, The University of Sydney, Sydney, NSW 2006, \\ Australia; ${ }^{5}$ Analytic and Clinical Cooperative Laboratory for Integrative Medicine, USYD \& CUHK, Sydney, NSW 2006, Australia; ${ }^{6}$ China \\ Academy of Chinese Medical Sciences, Beijing 100700, China
}

(C) Higher Education Press 2020

Abstract Coronavirus disease 2019 (COVID-19) is now pandemic worldwide and has heavily overloaded hospitals in Wuhan City, China during the time between late January and February. We reported the clinical features and therapeutic characteristics of moderate COVID-19 cases in Wuhan that were treated via the integration of traditional Chinese medicine (TCM) and Western medicine. We collected electronic medical record (EMR) data, which included the full clinical profiles of patients, from a designated TCM hospital in Wuhan. The structured data of symptoms and drugs from admission notes were obtained through an information extraction process. Other key clinical entities were also confirmed and normalized to obtain information on the diagnosis, clinical treatments, laboratory tests, and outcomes of the patients. A total of 293 COVID-19 inpatient cases, including 207 moderate and $86(29.3 \%)$ severe cases, were included in our research. Among these cases, 238 were discharged, 31 were transferred, and 24 (all severe cases) died in the hospital. Our COVID-19 cases involved elderly patients with advanced ages (57 years on average) and high comorbidity rates (61\%). Our results reconfirmed several well-recognized risk factors, such as age, gender (male), and comorbidities, as well as provided novel laboratory indications (e.g., cholesterol) and TCM-specific phenotype markers (e.g., dull tongue) that were relevant to COVID-19 infections and prognosis. In addition to antiviral/antibiotics and standard supportive therapies, TCM herbal prescriptions incorporating 290 distinct herbs were used in $273(93 \%)$ cases. The cases that received TCM treatment had lower death rates than those that did not receive TCM treatment $(17 / 273=6.2 \% \mathrm{vs}$. $7 / 20=35 \%, P=0.0004$ for all cases; $17 / 77=22 \%$ vs. $7 / 9=77.7 \%, P=0.002$ for severe cases). The TCM herbal prescriptions used for the treatment of COVID-19 infections mainly consisted of Pericarpium Citri Reticulatae, Radix Scutellariae, Rhizoma Pinellia, and their combinations, which reflected the practical TCM principles (e.g., clearing heat and dampening phlegm). Lastly, $59 \%$ of the patients received treatment, including antiviral, antibiotics, and Chinese patent medicine, before admission. This situation might have some effects on symptoms, such as fever and dry cough. By using EMR data, we described the clinical features and therapeutic characteristics of 293 COVID-19 cases treated via the integration of TCM herbal prescriptions and Western medicine. Clinical manifestations and treatments before admission and in the hospital were investigated. Our results preliminarily showed the potential effectiveness of TCM herbal prescriptions and their regularities in COVID-19 treatment.

Keywords COVID-19; traditional Chinese medicine; clinical features

Received May 7, 2019; accepted May 29, 2020

Correspondence: Josiah Poon, josiah.poon@sydney.edu.au;

Baoyan Liu, liuby5505@139.com;

Xuezhong Zhou, xzzhou@bjtu.edu.cn;

Xiaodong Li, lixiaodong555@126.com

${ }^{*}$ These authors contributed equally to this work.

\section{Introduction}

Coronavirus disease 2019 (COVID-19), which is caused by SARS-CoV-2 infection, has now become pandemic worldwide with more than ten millions confirmed cases. 
Similar to those of the common cold and flu, the symptoms of COVID-19 are fever and cough. Most (over 80\%) COVID-19 cases are moderate with good outcomes [1]. However, patients with critical cases of COVID-19 experience difficulty breathing. Cases with acute respiratory distress syndrome (ARDS) and multiple organ failure tend to have high mortality rates [2-4] because of the lack of effective therapies with antiviral drugs that are exclusive for COVID-19. In China, in addition to supportive treatment with Western medicine (e.g., antivirals), patients with COVID-19 receive personalized herbal prescriptions that are derived from traditional Chinese medicine (TCM). This combined treatment approach has been applied in $80 \%$ of COVID-19 cases and contributed to the low mortality rate in most cities outside of Hubei Province in China [5].

Prior studies on the treatment of COVID-19 with a combination of TCM herbal prescriptions and Western medicine have mainly involved less than 100 confirmed cases [6,7]. Conducting similar research with a moderate scale of observed clinical data to obtain results with increased reliability is necessary. We collected 293 retrospective COVID-19 cases in the form of electronic medical records (EMRs) from the largest TCM hospital in Wuhan, Hubei Provincial Hospital of Traditional Chinese Medicine. The values in structured data fields (e.g., demographic information, diagnoses, clinical orders, and laboratory tests) in the EMRs were normalized. TCM clinical named entities, such as symptoms and patent medicines, were extracted from admission records and discharge records by using text mining methods. We developed an information extraction system for extracting the various expressions of phenotype terms in clinical text by using a framework of human-machine collaboration strategies. This approach assured the data quality of the final extracted named entities.

This retrospective observation study described the epidemiologic features, clinical manifestations, treatments, and outcomes of 293 COVID-19 cases treated with combined TCM and Western medicine therapies.

\section{Materials and methods}

\section{Data collection}

This single-center retrospective study was performed at Hubei Provincial Hospital of Traditional Chinese Medicine. The EMRs (e.g., epidemiological, symptoms, and diagnoses), laboratory test results, and radiology reports of all inpatients in the pulmonary disease department from January 15, 2020 to March 2, 2020 were collected. In accordance with the diagnostic criteria of the fifth edition of the COVID-19 diagnosis and treatment plan [8], 26 patients who were still hospitalized and 38 inpatients without CT radiology results for COVID-19 were excluded, leaving 293 inpatients in the final analysis (Fig. 1). Among the 293 cases, only 191 were positive for SARS-CoV-2 nucleic acid, and 102 cases lacked this information. However, suspected cases with the characteristic manifestations of COVID-19 (e.g., patchy groundglass opacities, nodules, and patchy exudation) on CT scans were also treated as clinically confirmed cases even though their nucleic acid test results were negative (i.e., clinically diagnosed cases). As a result, 102 patients were also considered as positive cases. Under the guidance of clinical classification, these 293 patients were then divided into the moderate (including mild and moderate cases) and severe groups (including severe and critical cases) [9].

\section{Information extraction of text records from EMR data}

For investigating the trajectories of clinical manifestations and any prior treatments before admissions, we developed a clinical information extraction [10] tool to extract the various expressions of symptoms, mentions of drugs and therapies, and chronic diseases that were recorded in the medical histories of patients with COVID-19 from EMRs. Our information-extraction-annotation system used a framework based on the collaboration of dictionarybased annotation with manual verification strategies. This framework helped us fully extract the various expressions of phenotype terms in the clinical text and assure the data quality of the final extracted named entities. We typically extracted TCM clinical named entities, such as symptoms, drugs, and TCM patent medicines, within longitudinal events from admission records. Extraction would help us detect the longitudinal patterns of TCM symptoms and treatments for COVID-19.

\section{Data normalization}

Data standardization and normalization are required given that in EMRs, most data values are expressed in fine granularities to maintain an accurate profile of clinical entities. We normalized disease descriptions with the help of the International Classification of Disease Codes, Ninth Revision [11] and constrained symptom manifestations on the basis of the taxonomy of Symptom Ontology [12]. We finally identified 132 comorbid diseases with 13 systems and 99 symptoms in 8 symptom categories in this dataset. By using similar manual processing steps, we obtained 29 distinct terms of manifestations of the pulse and tongue, 12 terms of properties and flavors (e.g., bitter), 12 terms of channel tropism (e.g., stomach channel), and 254 terms of efficacy (e.g., antiviral) for TCM herbs and 35 drug categories of conventional therapies. All the admission data of the patients were verified and standardized by 




Fig. 1 Data filtering and grouping flow chart. COVID-19, coronavirus disease 2019; TCM, traditional Chinese medicine.

trained medical researchers to ensure highly accurate terminological mappings.

\section{Statistical and data analysis}

Descriptive and continuous variables were expressed as mean $\pm \mathrm{SD}$ or median (IQR) and subjected to MannWhitney U test. Categorical variables were expressed numerically as percentages and compared via $\chi^{2}$ test or Fisher's exact test between different groups. A two-sided $\alpha$ of less than 0.05 was considered statistically significant. All calculations and regression analysis were carried out by using SciPy and Statsmodels, which are a Python-based ecosystem of open-source software for mathematics, science, and engineering. Given that multiple herbs are typically found in a TCM prescription, we used the association rule mining algorithm (i.e., Apriori) [13] to obtain high-frequency herb combinations from prescriptions. Furthermore, we applied complex network analysis $[14,15]$ to investigate the core herb combinations that had high coverage in clinical prescriptions.

\section{Results}

\section{Clinical features of 293 relevant inpatients with COVID-19}

Among 293 cases, 191 were confirmed cases (65\%) that were positive for SARS-CoV-2 nucleic acid and 102 were clinically diagnosed cases $(35 \%)$ with confirmed positive chest CT reports. A total of $273(93 \%)$ patients received integrated TCM and Western medicine therapies. In this study, 24 cases died during hospitalization, 238 cases were discharged, and 31 cases were transferred to other hospitals (e.g., Jinyintan Hospital). The average age of these patients was 57 years. Among the patients, 54\% were female and $46 \%$ were male. Severe cases had longer average hospital stays (15.5 days vs. 14 days) and shorter symptom onset time ( 9 days vs. 11.5 days) than moderate cases. A total of $178(61 \%)$ patients had disease comorbidities, particularly diseases of the metabolic and circulatory system. Hypertension was the most common comorbidity (24\%), followed by diabetes (16\%) and coronary heart disease 
(6\%). Severe cases tended to have a higher degree of comorbidities than moderate cases. Approximately $83 \%$ of patients had manifestations of the respiratory tract. Coughing was the most prevalent symptom (51\%), and nearly half of the cases suffered from digestive and neurological system discomfort, such as loss of appetite and insomnia. In addition, chest tightness, wheezing, and breathing difficulty might indicate severe conditions. Although their overall mean values were within the normal range, severe cases tended to have higher frequencies and values of breath and blood pressure than moderate cases. This observation was similar between the survivors and nonsurvivors (Tables 1 and 2). For TCM-specific manifestations, a high number of cases manifested yellow fur $(57 \%)$ and reddish tongue (53\%), and a higher number of patients with severe cases exhibited dull tongue and rapid pulse, which usually indicates the heat properties of the coronavirus from the pathological perspective of TCM. Similar results were also reflected in TCM syndromes, such as the syndrome of phlegm-heat lung obstruction.
Although most patients with COVID-19 had normal white blood cell and neutrophil counts at the beginning of the disease, they tended to have abnormal lymphocyte counts and elevated C-reactive protein and IL-6 levels, indicating the possibility of secondary infection. These parameters slightly returned to normal after treatment. In addition, myoglobin, bilirubin, and $\gamma$-GT levels were clearly higher in severe cases than in moderate cases. Notably, triglyceride and total cholesterol levels were higher in moderate cases than in severe cases $(1.6 \mathrm{mmol} / \mathrm{L}$ vs. $1.4 \mathrm{mmol} / \mathrm{L}$ and $4.4 \mathrm{mmol} / \mathrm{L}$ vs. $3.8 \mathrm{mmol} / \mathrm{L}$ ). The ratio of partial of oxygen saturation was significantly lower in severe cases than in moderate cases, implying an increased likelihood of developing ARDS and receiving mechanical ventilation (Table 3 ).

Next, we fitted clinical outcomes through multivariate logistic regression (discharged $=0$, transferred $=2$, and died $=4$ ) by using demographic features; clinical classification; and the clinical manifestations of symptoms, comorbidities, and TCM syndromes with $>10$ frequency.

Table 1 Characteristics of moderate and severe COVID-19 cases

\begin{tabular}{|c|c|c|c|c|}
\hline Demographics and clinical characteristics & $\begin{array}{l}\text { Total } \\
\quad(n=293)\end{array}$ & $\begin{array}{l}\text { Moderate } \\
(n=207)\end{array}$ & $\begin{array}{l}\text { Severe } \\
(n=86)\end{array}$ & $P$ values \\
\hline Age (year) & $57.1 \pm 15.6$ & $54.0 \pm 15.0$ & $64.6 \pm 14.5$ & $1.25 \mathrm{E}-07^{* *}$ \\
\hline Sex & & & & $2.50 \mathrm{E}-05^{* *}$ \\
\hline Female & $158(54 \%)$ & $128(62 \%)$ & $30(35 \%)$ & \\
\hline Male & $135(46 \%)$ & $79(38 \%)$ & $56(65 \%)$ & \\
\hline Hospital stay duration (day) & $14.5 \pm 6.1$ & $14.0 \pm 5.1$ & $15.5 \pm 7.9$ & $5.14 \mathrm{E}-02$ \\
\hline Onset time (day) & $10.8 \pm 7.2$ & $11.5 \pm 7.5$ & $9.1 \pm 6.3$ & $1.58 \mathrm{E}-02^{*}$ \\
\hline Any comorbidity & $178(61 \%)$ & $108(52 \%)$ & $70(81 \%)$ & $3.09 \mathrm{E}-06^{* *}$ \\
\hline Endocrine and metabolic diseases & $101(34 \%)$ & $58(28 \%)$ & $43(50 \%)$ & $3.12 \mathrm{E}-04^{* *}$ \\
\hline Diabetes mellitus & $47(16 \%)$ & $25(12 \%)$ & $22(26 \%)$ & $4.13 \mathrm{E}-03^{* *}$ \\
\hline Circulatory system diseases & $91(31 \%)$ & $47(23 \%)$ & $44(51 \%)$ & $1.64 \mathrm{E}-06^{* *}$ \\
\hline Hypertension & $69(24 \%)$ & $40(19 \%)$ & $29(34 \%)$ & $8.17 \mathrm{E}-03^{* *}$ \\
\hline Coronary heart disease & $19(6 \%)$ & $8(4 \%)$ & $11(13 \%)$ & $4.72 \mathrm{E}-03^{* *}$ \\
\hline Cerebral infarction & $11(4 \%)$ & $2(1 \%)$ & $9(10 \%)$ & $3.73 \mathrm{E}-04^{* *}$ \\
\hline Digestive system diseases & $39(13 \%)$ & $13(6 \%)$ & $26(30 \%)$ & $3.88 \mathrm{E}-08^{* *}$ \\
\hline Chronic gastritis & $5(2 \%)$ & $1(0.5 \%)$ & $4(5 \%)$ & $4.41 \mathrm{E}-02^{*}$ \\
\hline Genital system diseases & $22(8 \%)$ & $11(5 \%)$ & $11(13 \%)$ & $2.70 \mathrm{E}-02^{*}$ \\
\hline Chronic kidney disease & $4(1 \%)$ & $0(0 \%)$ & $4(5 \%)$ & $1.01 \mathrm{E}-02^{*}$ \\
\hline \multicolumn{5}{|l|}{ Signs and symptoms } \\
\hline Respiratory system symptoms & $243(83 \%)$ & $171(83 \%)$ & $72(84 \%)$ & $8.18 \mathrm{E}-01$ \\
\hline Coughing & $148(51 \%)$ & $101(49 \%)$ & $47(55 \%)$ & $3.61 \mathrm{E}-01$ \\
\hline Chest tightness & $104(35 \%)$ & $61(29 \%)$ & $43(50 \%)$ & $8.24 \mathrm{E}-04^{* *}$ \\
\hline Wheezing & $104(35 \%)$ & $59(29 \%)$ & $45(52 \%)$ & $1.04 \mathrm{E}-04^{* *}$ \\
\hline Respiratory difficulty & $34(12 \%)$ & $14(7 \%)$ & $20(23 \%)$ & $5.97 \mathrm{E}-05^{* *}$ \\
\hline General symptoms & $181(62 \%)$ & $121(58 \%)$ & $60(70 \%)$ & $8.18 \mathrm{E}-01$ \\
\hline Fever & $102(35 \%)$ & $57(28 \%)$ & $45(52 \%)$ & $4.99 \mathrm{E}-05^{* *}$ \\
\hline Digestive system symptoms & $138(47 \%)$ & $86(42 \%)$ & $52(60 \%)$ & $3.13 \mathrm{E}-03^{* *}$ \\
\hline Reduced appetite & $87(30 \%)$ & $52(25 \%)$ & $35(41 \%)$ & $7.87 \mathrm{E}-03^{* *}$ \\
\hline Nervous system symptoms & $120(41 \%)$ & $75(36 \%)$ & $45(52 \%)$ & $1.07 \mathrm{E}-02^{*}$ \\
\hline Insomnia & $96(33 \%)$ & $56(27 \%)$ & $40(47 \%)$ & $7.87 \mathrm{E}-03^{* *}$ \\
\hline Circulatory system symptoms & $24(8 \%)$ & $12(6 \%)$ & $12(14 \%)$ & $2.04 \mathrm{E}-02^{*}$ \\
\hline
\end{tabular}




\begin{tabular}{|c|c|c|c|c|}
\hline \multicolumn{5}{|c|}{ (Continued) } \\
\hline Demographics and clinical characteristics & $\begin{array}{l}\text { Total } \\
\quad(n=293)\end{array}$ & $\begin{array}{l}\text { Moderate } \\
\quad(n=207)\end{array}$ & $\begin{array}{l}\text { Severe } \\
\quad(n=86)\end{array}$ & $P$ values \\
\hline Palpitations & $24(8 \%)$ & $12(6 \%)$ & $12(14 \%)$ & $2.04 \mathrm{E}-02^{*}$ \\
\hline Temperature $\left({ }^{\circ} \mathrm{C}\right)$ & $36.9 \pm 0.7$ & $36.8 \pm 0.7$ & $37.0 \pm 0.8$ & $1.03 \mathrm{E}-01$ \\
\hline Breathing & $20.9 \pm 4.1$ & $20.3 \pm 2.7$ & $22.2 \pm 5.9$ & $9.25 \mathrm{E}-04^{* *}$ \\
\hline \multicolumn{5}{|l|}{ Blood pressure } \\
\hline Systolic pressure (mmHG) & $126.3 \pm 16.4$ & $123.0 \pm 13.7$ & $132.9 \pm 19.2$ & $1.99 \mathrm{E}-06^{* *}$ \\
\hline Diastolic pressure (mmHG) & $75.9 \pm 10.3$ & $75.0 \pm 9.3$ & $77.7 \pm 11.9$ & $2.05 \mathrm{E}-02^{*}$ \\
\hline \multicolumn{5}{|l|}{ Tongue fur and pulse manifestations } \\
\hline Reddish tongue & $155(53 \%)$ & $112(54 \%)$ & $43(50 \%)$ & $5.21 \mathrm{E}-01$ \\
\hline Dull tongue & $12(4 \%)$ & $5(2 \%)$ & $7(8 \%)$ & $2.44 \mathrm{E}-02^{*}$ \\
\hline Yellow fur & $167(57 \%)$ & $125(60 \%)$ & $42(49 \%)$ & $6.90 \mathrm{E}-02$ \\
\hline Greasy fur & $73(25 \%)$ & $52(25 \%)$ & $21(24 \%)$ & $8.99 \mathrm{E}-01$ \\
\hline Rapid pulse & $42(14 \%)$ & $22(11 \%)$ & $20(23 \%)$ & $4.97 \mathrm{E}-03^{* *}$ \\
\hline \multicolumn{5}{|l|}{ TCM syndromes } \\
\hline Syndrome of pathogenic qi lung invasion & $91(31 \%)$ & $56(27 \%)$ & $35(41 \%)$ & $2.15 \mathrm{E}-02^{*}$ \\
\hline Syndrome of phlegm-heat lung obstruction & $84(29 \%)$ & $62(30 \%)$ & $22(26 \%)$ & $4.51 \mathrm{E}-01$ \\
\hline Syndrome of stagnant and jamming wind-heat & $33(11 \%)$ & $26(13 \%)$ & $7(8 \%)$ & $2.76 \mathrm{E}-01$ \\
\hline Syndrome of wind-heat weifen invasion & $18(6 \%)$ & $13(6 \%)$ & $5(6 \%)$ & $8.80 \mathrm{E}-01$ \\
\hline Syndrome of phlegm-dampness lung obstruction & $14(5 \%)$ & $10(5 \%)$ & $4(5 \%)$ & $8.14 \mathrm{E}-01$ \\
\hline \multicolumn{5}{|l|}{ Laboratory findings } \\
\hline WBC $\left((3.5-9.5) \times 10^{9} / \mathrm{L}\right)$ & $5.0(3.9-7.0)$ & $4.9(3.9-6.2)$ & $5.8(3.9-8.0)$ & $7.14 \mathrm{E}-02$ \\
\hline Neutrophil count $\left((1.8-6.3) \times 10^{9} / \mathrm{L}\right)$ & $3.5(2.6-5.3)$ & $3.4(2.5-4.2)$ & $4.6(3.1-6.6)$ & $1.55 \mathrm{E}-03^{* *}$ \\
\hline Lymphocyte count $\left((1.1-3.2) \times 10^{9} / \mathrm{L}\right)$ & $1.0(0.7-1.4)$ & $1.2(0.9-1.6)$ & $0.7(0.5-1.0)$ & $6.76 \mathrm{E}-09^{* *}$ \\
\hline C-reactive protein $(<3 \mathrm{mg} / \mathrm{L})$ & $18.2(4.2-63.5)$ & $8.1(2.3-25.4)$ & $58.3(22-117.5)$ & $1.89 \mathrm{E}-12^{* *}$ \\
\hline Triglycerides $(<1.7 \mathrm{mmol} / \mathrm{L})$ & $1.6(1.1-2.0)$ & $1.6(1.2-2.1)$ & $1.4(1.1-1.79)$ & $3.71 \mathrm{E}-02^{*}$ \\
\hline Myoglobin $(<100 \mathrm{ng} / \mathrm{mL})$ & $54.0(35.3-91.5)$ & $43.6(35.3-58.4)$ & $76(45.7-147.1)$ & $4.88 \mathrm{E}-03^{* *}$ \\
\hline Total bilirubin $(<21 \mu \mathrm{mol} / \mathrm{L})$ & $8.0(6.2-11.6)$ & $7.2(5.8-9.7)$ & $10.3(7.2-13.6)$ & $6.97 \mathrm{E}-05^{* *}$ \\
\hline Total cholesterol $(<5.17 \mathrm{mmol} / \mathrm{L})$ & $4.2(3.5-4.9)$ & $4.4(3.9-5.0)$ & $3.8(3.4-4.4)$ & $2.17 \mathrm{E}-03^{* *}$ \\
\hline $\operatorname{LDL}(<3.4 \mathrm{mmol} / \mathrm{L})$ & $2.2(1.8-2.6)$ & $2.3(1.9-2.8)$ & $2.0(1.7-2.5)$ & $1.47 \mathrm{E}-02^{*}$ \\
\hline HDL $(1.29-1.55 \mathrm{mmol} / \mathrm{L})$ & $1.1(0.9-1.2)$ & $1.1(0.9-1.2)$ & $1.0(0.9-1.2)$ & $5.38 \mathrm{E}-02$ \\
\hline $\operatorname{ALT}(13-40 \mathrm{U} / \mathrm{L})$ & $22.0(18.0-34.0)$ & $20.0(17.0-27.0)$ & $33.0(22.0-49.0)$ & $8.18 \mathrm{E}-07^{*}$ \\
\hline$\gamma$-GT $(7-45 \mathrm{U} / \mathrm{L})$ & $23.0(15.0-40.0)$ & $22.0(14.3-35.8)$ & $26.5(17.0-47.5)$ & $5.54 \mathrm{E}-02$ \\
\hline Uric acid $(155-357 \mu \mathrm{mol} / \mathrm{L})$ & $300(234.5-369)$ & $315(241-364.5)$ & $285(231.5-396)$ & $7.66 \mathrm{E}-01$ \\
\hline Platelet count $\left((125-350) \times 10^{9} / \mathrm{L}\right)$ & $210(163.3-253)$ & $228(171-267)$ & $190(140-240)$ & $8.96 \mathrm{E}-03^{* *}$ \\
\hline Prothrombin time $(9.9-12.9 \mathrm{~s})$ & $12.1(11.5-12.6)$ & $11.9(11.4-12.4)$ & $12.5(11.9-13.6)$ & $1.33 \mathrm{E}-04^{* *}$ \\
\hline Oxygen saturation $(96 \%-98 \%)$ & $97.1(94.9-98.7)$ & $97.8(97.0-99.0)$ & $96.1(93.3-98.7)$ & $4.39 \mathrm{E}-03^{* *}$ \\
\hline Interleukin-6 $(<7 \mathrm{pg} / \mathrm{mL})$ & $14.6(4.4-39.5)$ & $9.8(2.5-18.7)$ & $34.2(13.7-87.9)$ & $9.95 \mathrm{E}-05^{* *}$ \\
\hline
\end{tabular}

Data are presented as mean \pm SD or median (IQR), $n(\%)$, or $n / N(\%)$, where $N$ is the total number of patients in a group. $P$ values were calculated via MannWhitney U test, $\chi^{2}$ test, or Fisher's exact test as appropriate. $P$ values were used to compare moderate and severe groups. Onset time means the earliest record of symptoms before admission. WBC, white blood cell count; ALT, aspartate aminotransferase; $\gamma$-GT, $\gamma$-glutamyltransferase; LDL, low-density lipoprotein; HDL, high-density lipoprotein. $* P<0.05, * * P<0.01$.

We found that the fitted model could explain $51.7 \%$ of variance of the clinical outcomes. Several significant associations were detected (Table 4). The risk factors for death included advanced age (coefficient $=0.753, P=$ 0.002 ); gender $($ female $=1$, male $=2$; coefficient $=0.173, P$ $=0.042$ ); severe disease condition (coefficient $=0.856$, $P<0.0001)$; and clinical manifestations, including coughing up of phlegm (coefficient $=0.329, P=0.006$ ) and cerebral infarction (coefficient $=0.758, P=0.001$ ). We also found that patients with fatigue (coefficient $=-0.23, P$ $=0.01$ ) and yellow fur (coefficient $=-0.31, P=0.042$ ) were more likely to be discharged than to be deceased.

\section{Therapeutic characteristics of Western medicine and TCM patent prescriptions}

Western medicine with antibacterial and antiviral agents remained the first choices for the treatment of the 293 inpatient cases (Tables 5 and 6). Specifically, 258 (88\%) patients received antibacterial agents, $247(84 \%)$ patients received antiviral agents, and $135(46 \%)$ patients received glucocorticoids. Arbidol was given to 199 (68\%) patients, ribavirin to $56(19 \%)$ patients, and interferon to $52(18 \%)$ patients. Large amounts of hormones (e.g., methylprednisolone), digestive system drugs (e.g., omeprazole), and 
Table 2 Characteristics based on the prognosis of severe cases

\begin{tabular}{|c|c|c|c|c|}
\hline Demographics and clinical characteristics & $\begin{array}{l}\text { Discharged } \\
(n=40)\end{array}$ & $\begin{array}{l}\text { Transferred } \\
(n=22)\end{array}$ & $\begin{array}{l}\text { Died } \\
\quad(n=24)\end{array}$ & $P$ values \\
\hline Age (year) & $58.3 \pm 13.5$ & $66.3 \pm 13.8$ & $73.6 \pm 11.8$ & $1.02 \mathrm{E}-04^{* *}$ \\
\hline Sex & & & & $1.25 \mathrm{E}-02^{*}$ \\
\hline Female & $21(53 \%)$ & $4(18 \%)$ & $5(21 \%)$ & \\
\hline Male & $19(48 \%)$ & $18(82 \%)$ & $19(79 \%)$ & \\
\hline Hospital stay duration (day) & $17.7 \pm 5.3$ & $15.5 \pm 9.8$ & $11.9 \pm 8.6$ & $2.03 \mathrm{E}-03^{* *}$ \\
\hline Onset time (day) & $10.9 \pm 6.3$ & $7.6 \pm 5.8$ & $7.7 \pm 6.5$ & $8.91 \mathrm{E}-02$ \\
\hline Any comorbidity & $33(83 \%)$ & $16(73 \%)$ & $21(88 \%)$ & $8.59 \mathrm{E}-01$ \\
\hline Hypertension & $13(33 \%)$ & $8(37 \%)$ & $8(33 \%)$ & $9.45 \mathrm{E}-01$ \\
\hline Diabetes mellitus & $11(28 \%)$ & $4(18 \%)$ & $7(29 \%)$ & $8.86 \mathrm{E}-01$ \\
\hline Coronary heart disease & $4(10 \%)$ & $3(14 \%)$ & $4(17 \%)$ & $6.96 \mathrm{E}-01$ \\
\hline Acute coronary syndrome & $0(0 \%)$ & $0(0 \%)$ & $3(13 \%)$ & $9.30 \mathrm{E}-02$ \\
\hline Cerebral infarction & $3(8 \%)$ & $1(5 \%)$ & $5(21 \%)$ & $2.42 \mathrm{E}-01$ \\
\hline \multicolumn{5}{|l|}{ Complications } \\
\hline Respiratory failure & $6(15 \%)$ & $3(14 \%)$ & $11(46 \%)$ & $6.86 \mathrm{E}-03^{* *}$ \\
\hline \multicolumn{5}{|l|}{ Signs and symptoms } \\
\hline Coughing & $22(55 \%)$ & $12(55 \%)$ & $13(54 \%)$ & $9.48 \mathrm{E}-01$ \\
\hline Wheezing & $27(68 \%)$ & $6(27 \%)$ & $12(50 \%)$ & $1.65 \mathrm{E}-01$ \\
\hline Fever & $24(60 \%)$ & $11(50 \%)$ & $10(42 \%)$ & $1.55 \mathrm{E}-01$ \\
\hline Chest tightness & $21(53 \%)$ & $10(45 \%)$ & $12(50 \%)$ & $8.46 \mathrm{E}-01$ \\
\hline Insomnia & $19(48 \%)$ & $10(45 \%)$ & $11(46 \%)$ & $8.97 \mathrm{E}-01$ \\
\hline Reduced appetite & $19(48 \%)$ & $9(41 \%)$ & $7(29 \%)$ & $1.48 \mathrm{E}-01$ \\
\hline Respiratory difficulty & $8(20 \%)$ & $7(32 \%)$ & $5(21 \%)$ & $9.36 \mathrm{E}-01$ \\
\hline Temperature $\left({ }^{\circ} \mathrm{C}\right)$ & $37.0 \pm 0.8$ & $37.1 \pm 0.8$ & $37.0 \pm 0.8$ & $8.84 \mathrm{E}-01$ \\
\hline Breathing & $21.0 \pm 5.3$ & $22.8 \pm 4.9$ & $23.3 \pm 7.5$ & $9.83 \mathrm{E}-02$ \\
\hline \multicolumn{5}{|l|}{ Blood pressure } \\
\hline Systolic pressure (mmHG) & $129.0 \pm 18.3$ & $129.5 \pm 14.8$ & $142.0 \pm 22.4$ & $5.56 \mathrm{E}-02$ \\
\hline Diastolic pressure (mmHG) & $76.0 \pm 11.9$ & $78.6 \pm 10.1$ & $79.4 \pm 13.5$ & $1.89 \mathrm{E}-01$ \\
\hline \multicolumn{5}{|l|}{ Tongue fur and pulse manifestations } \\
\hline Reddish tongue & $22(55 \%)$ & $12(55 \%)$ & $9(38 \%)$ & $1.75 \mathrm{E}-01$ \\
\hline Yellow fur & $22(55 \%)$ & $13(59 \%)$ & $7(29 \%)$ & $4.44 \mathrm{E}-02^{*}$ \\
\hline Stringy pulse & $12(30 \%)$ & $6(27 \%)$ & $4(17 \%)$ & $3.71 \mathrm{E}-01$ \\
\hline Greasy fur & $13(33 \%)$ & $6(27 \%)$ & $2(8 \%)$ & $5.68 \mathrm{E}-02$ \\
\hline \multicolumn{5}{|l|}{ TCM syndromes } \\
\hline Syndrome of pathogenic qi lung invasion & $16(40 \%)$ & $13(59 \%)$ & $6(25 \%)$ & $2.21 \mathrm{E}-01$ \\
\hline Syndrome of phlegm-heat lung obstruction & $15(38 \%)$ & $2(9 \%)$ & $5(21 \%)$ & $1.64 \mathrm{E}-01$ \\
\hline
\end{tabular}

Data are presented as mean $\pm \mathrm{SD}$ or median (IQR), $n(\%)$, or $n / N(\%)$, where $N$ is the total number of patients in a group. $P$ values were calculated via MannWhitney U test, $\chi^{2}$ test, or Fisher's exact test as appropriate. $P$ values were used to compare the discharge and death groups. Onset time means the earliest record of symptoms before admission. * $P<0.05$, ** $P<0.01$.

biological products (e.g., albumin) were used in severe cases. In particular, large amounts of antishock drugs were used in the rescue phase of critical cases. However, except for a typical antiviral drug (i.e., arbidol), most other drugs were used for severe cases instead of for moderate cases. The use of some drugs, such as imipenem and cilastatin sodium, significantly differed between the two groups.

\section{Therapeutic characteristics of TCM herbal prescriptions}

A total of $20(6.8 \%)$ patients did not receive Chinese herbal medicine, among whom 2 were in critical condition and not allowed to receive TCM therapy. EMR notes indicated that the remaining 18 cases (11 moderate and 7 severe) refused to take Chinese herbal medicine. The hospital stays of these 18 patients ranged from 5 days to 28 days. A total of $273(93 \%)$ patients received 1176 herbal prescriptions (Table 7). Each prescription was prepared as a compound therapy that consisted of 16-18 herbs (Fig. 2A). A total of $256(>95 \%)$ of the 269 survivors who were discharged or transferred received herbal prescriptions, whereas only 17 $(71 \%)$ of the 24 deceased received herbal prescriptions instead (Table 7). 
Table 3 Laboratory findings based on the prognosis of severe cases before and after hospitalization treatment

\begin{tabular}{lllll}
\hline Laboratory findings & Test time & $\begin{array}{c}\text { Discharged } \\
(n=40)\end{array}$ & $\begin{array}{c}\text { Transferred } \\
(n=22)\end{array}$ & $\begin{array}{c}\text { Died } \\
(n=24)\end{array}$ \\
\hline WBC $\left((3.5-9.5) \times 10^{9} / \mathrm{L}\right)$ & First & $5.5(4.5-7.7)$ & $6.5(3.8-7.6)$ & $6.3(3.4-8.8)$ \\
& Last & $7.6(4.4-8.8)$ & $8.1(5.0-12.7)$ & $11.9(6.9-12.7)$ \\
Neutrophil count $\left((1.8-6.3) \times 10^{9} / \mathrm{L}\right)$ & $P$ values & $2.98 \mathrm{E}-01$ & $3.96 \mathrm{E}-01$ & $4.60 \mathrm{E}-02$ \\
& First & $4.1(3.2-6.4)$ & $5.4(3.2-6.1)$ & $4.4(2.6-8.5)$ \\
& Last & $5.4(3.1-6.9)$ & $6.1(3.7-11.3)$ & $10.3(4.9-12.1)$ \\
C-reactive protein $(<3 \mathrm{mg} / \mathrm{L})$ & $P$ values & $6.50 \mathrm{E}-01$ & $5.27 \mathrm{E}-01$ & $6.43 \mathrm{E}-02$ \\
& First & $45.1(19.8-87.2)$ & $37.5(16.2-115.8)$ & $81.6(51-152.9)$ \\
Total cholesterol $(<5.17 \mathrm{mmol} / \mathrm{L})$ & Last & $3.1(1.1-12.4)$ & $34.60(12.15-99.2)$ & $72.3(33-144.3)$ \\
& $P$ values & $2.99 \mathrm{E}-04 * *$ & $6.85 \mathrm{E}-01$ \\
HDL $(1.29-1.55 \mathrm{mmol} / \mathrm{L})$ & First & $4.1(3.4-4.4)$ & $3.5(3.4-4.0)$ & $4.0(3.5-4.9)$ \\
& Last & $5.6(3.1-5.8)$ & $3.7(3.3-4.4)$ & $3.4(3.0-4.1)$ \\
& $P$ values & $3.89 \mathrm{E}-01$ & $9.50 \mathrm{E}-01$ & $5.12 \mathrm{E}-02$ \\
& First & $0.99(0.93-1.15)$ & $0.95(0.79-1.17)$ & $1.01(0.91-1.10)$ \\
& Last & $0.95(0.94-0.96)$ & $0.90(0.89-0.91)$ & $0.77(0.56-0.94)$ \\
\hline
\end{tabular}

Data are presented as median (IQR). $P$ values were calculated via Mann-Whitney $\mathrm{U}$ test and used to compare the initial and final laboratory test results of each group during the hospitalization period. WBC, white blood cell count; HDL, high-density lipoprotein. ${ }^{*} P<0.05,{ }^{*} P<0.01$.

Table 4 Associated clinical features for the prognosis of COVID-19

\begin{tabular}{|c|c|c|c|c|}
\hline Variable & Coefficient & $P$ values & $\mathrm{LL}$ & UL \\
\hline \multicolumn{5}{|l|}{ Baseline variables } \\
\hline Age (year) & 0.753 & $0.002 * *$ & 0.286 & 1.221 \\
\hline Gender (female/male) & 0.173 & $0.042 *$ & 0.006 & 0.341 \\
\hline Clinical classification (moderate/severe) & 0.856 & $<0.0001^{* *}$ & 0.647 & 1.066 \\
\hline \multicolumn{5}{|l|}{ Clinical variables (yes/no) } \\
\hline Cough & -0.14 & 0.115 & -0.335 & 0.036 \\
\hline Chest tightness & 0.013 & 0.894 & -0.184 & 0.21 \\
\hline Wheezing & -0.06 & 0.525 & -0.266 & 0.136 \\
\hline Fever & -0.08 & 0.354 & -0.277 & 0.099 \\
\hline Fatigue & -0.23 & $0.01 *$ & -0.413 & -0.056 \\
\hline Insomnia & 0.038 & 0.718 & -0.173 & 0.251 \\
\hline Reduced appetite & -0.19 & 0.066 & -0.405 & 0.013 \\
\hline Coughing of phlegm & 0.329 & $0.006 * *$ & 0.094 & 0.564 \\
\hline Dry cough & -0.07 & 0.47 & -0.289 & 0.134 \\
\hline Respiratory difficulty & -0.02 & 0.873 & -0.294 & 0.25 \\
\hline Throat discomfort & -0.10 & 0.487 & -0.401 & 0.191 \\
\hline White sputum & 0.276 & 0.111 & -0.064 & 0.616 \\
\hline Headache & -0.13 & 0.429 & -0.463 & 0.197 \\
\hline Palpitation & -0.11 & 0.456 & -0.431 & 0.194 \\
\hline Diarrhea & 0.066 & 0.68 & -0.252 & 0.386 \\
\hline Loose stool & 0.053 & 0.765 & -0.296 & 0.402 \\
\hline Bitter taste in mouth & -0.24 & 0.303 & -0.698 & 0.218 \\
\hline Vertigo & -0.36 & 0.061 & -0.74 & 0.017 \\
\hline Dry mouth & 0.162 & 0.514 & -0.327 & 0.652 \\
\hline Limb pain & 0.222 & 0.283 & -0.185 & 0.631 \\
\hline Hypertension & -0.09 & 0.3 & -0.161 & 0.52 \\
\hline Diabetes mellitus & -0.06 & 0.393 & -0.309 & 0.122 \\
\hline Coronary heart disease & 0.179 & 0.584 & -0.301 & 0.17 \\
\hline Cerebral infarction & 0.758 & $0.001 * *$ & 0.308 & 1.209 \\
\hline Reddish tongue & -0.09 & 0.221 & -0.352 & 0.082 \\
\hline
\end{tabular}




\begin{tabular}{|c|c|c|c|c|}
\hline & & & & (Continued) \\
\hline $\begin{array}{l}\text { Variable } \\
\text {. }\end{array}$ & Coefficient & $P$ values & $\overline{L L}$ & $\overline{\mathrm{UL}}$ \\
\hline Pink tongue & -0.10 & 0.701 & -0.566 & 0.381 \\
\hline Deep red tongue & -0.04 & 0.608 & -0.252 & 0.43 \\
\hline Pale tongue & -0.09 & 0.929 & -0.263 & 0.24 \\
\hline Dull tongue & -0.11 & 0.619 & -0.446 & 0.266 \\
\hline Yellow fur & -0.31 & $0.042 * *$ & -0.616 & -0.011 \\
\hline Greasy fur & -0.13 & 0.29 & -0.113 & 0.376 \\
\hline Thin fur & -0.01 & 0.605 & -0.517 & 0.302 \\
\hline White fur & -0.26 & 0.529 & -0.301 & 0.155 \\
\hline Thick fur & -0.01 & 0.652 & -0.618 & 0.388 \\
\hline Wiry pulse & -0.07 & 0.087 & -0.573 & 0.039 \\
\hline Slippery pulse & 0.131 & 0.913 & -0.283 & 0.253 \\
\hline Rapid pulse & 0.155 & 0.248 & -0.109 & 0.42 \\
\hline Floating pulse & 0.088 & 0.093 & -0.046 & 0.592 \\
\hline Thready pulse & 0.273 & 0.841 & -0.517 & 0.421 \\
\hline Syndrome of pathogenic qi lung invasion & -0.26 & 0.946 & -0.415 & 0.444 \\
\hline Syndrome of phlegm-heat lung obstruction & -0.02 & $0.033^{*}$ & -0.501 & -0.021 \\
\hline Syndrome of stagnant and jamming wind-heat & -0.07 & 0.864 & -0.266 & 0.223 \\
\hline Syndrome of wind-heat weifen invasion & 0.014 & 0.925 & -0.445 & 0.405 \\
\hline
\end{tabular}

LL, lower limit; UL, upper limit; including all variables with more than 10 patients. $* P<0.05, * * P<0.01$.

Table 5 Clinical therapies for moderate and severe COVID-19 cases

\begin{tabular}{|c|c|c|c|c|}
\hline Medicine & Total $(n=293)$ & Moderate $(n=207)$ & Severe $(n=86)$ & $P$ values \\
\hline Antibiotics & $258(88 \%)$ & $175(85 \%)$ & $83(97 \%)$ & $7.83 \mathrm{E}-03^{* *}$ \\
\hline Moxifloxacin & $232(79 \%)$ & $152(73 \%)$ & $80(93 \%)$ & $1.69 \mathrm{E}-04 * *$ \\
\hline Cefoperazone & $91(31 \%)$ & $42(20 \%)$ & $49(57 \%)$ & $2.19 \mathrm{E}-09 * *$ \\
\hline Imipenem and cilastatin sodium & $49(17 \%)$ & $7(3 \%)$ & $42(49 \%)$ & $1.12 \mathrm{E}-19^{* *}$ \\
\hline Antivira & $247(84 \%)$ & $171(83 \%)$ & $76(88 \%)$ & $2.17 \mathrm{E}-01$ \\
\hline Arbidol & $199(68 \%)$ & $143(69 \%)$ & $56(65 \%)$ & $5.83 \mathrm{E}-01$ \\
\hline Ribavirin & $56(19 \%)$ & $38(18 \%)$ & $18(21 \%)$ & $6.27 \mathrm{E}-01$ \\
\hline Interferon & $52(18 \%)$ & $33(16 \%)$ & $19(22 \%)$ & $2.40 \mathrm{E}-01$ \\
\hline Digestive system drugs & $190(65 \%)$ & $117(57 \%)$ & $73(85 \%)$ & $3.65 \mathrm{E}-06^{* *}$ \\
\hline Omeprazole & $79(27 \%)$ & $42(20 \%)$ & $37(43 \%)$ & $1.42 \mathrm{E}-04 * *$ \\
\hline Pantoprazole & $78(27 \%)$ & $45(22 \%)$ & $33(38 \%)$ & $5.52 \mathrm{E}-03^{* *}$ \\
\hline TCM patent prescription & $184(63 \%)$ & $112(54 \%)$ & $72(84 \%)$ & $1.79 \mathrm{E}-06^{* *}$ \\
\hline Baicalin & $52(18 \%)$ & $19(9 \%)$ & $33(38 \%)$ & $1.59 \mathrm{E}-08^{* *}$ \\
\hline Xue Bi Jing injection & $46(16 \%)$ & $13(6 \%)$ & $33(38 \%)$ & $7.81 \mathrm{E}-11^{* *}$ \\
\hline Glucocorticoids & $135(46 \%)$ & $68(33 \%)$ & $67(78 \%)$ & $1.84 \mathrm{E}-12 * *$ \\
\hline Methylprednisolone & $116(40 \%)$ & $51(25 \%)$ & $65(76 \%)$ & $7.00 \mathrm{E}-16^{* *}$ \\
\hline Nutrition support & $134(46 \%)$ & $69(33 \%)$ & $65(76 \%)$ & $3.83 \mathrm{E}-11^{* *}$ \\
\hline Potassium chloride & $61(21 \%)$ & $28(14 \%)$ & $33(38 \%)$ & $5.79 \mathrm{E}-06^{* *}$ \\
\hline Biological products & $127(43 \%)$ & $64(31 \%)$ & $63(73 \%)$ & $2.75 \mathrm{E}-11 * *$ \\
\hline Human albumin & $61(21 \%)$ & $20(10 \%)$ & $41(48 \%)$ & $3.19 \mathrm{E}-12^{* *}$ \\
\hline Antitussive and antiasthmatic & $126(43 \%)$ & $74(36 \%)$ & $52(60 \%)$ & $9.97 \mathrm{E}-05^{* *}$ \\
\hline Ambroxol & $52(18 \%)$ & $23(11 \%)$ & $29(34 \%)$ & $1.55 \mathrm{E}-05^{* *}$ \\
\hline Vitamins & $111(38 \%)$ & $61(29 \%)$ & $50(58 \%)$ & $4.09 \mathrm{E}-06^{* *}$ \\
\hline Vitamin C & $68(23 \%)$ & $35(17 \%)$ & $33(38 \%)$ & $1.30 \mathrm{E}-04 * *$ \\
\hline Antipyretic analgesic & $84(29 \%)$ & $44(21 \%)$ & $40(47 \%)$ & $1.34 \mathrm{E}-05^{* *}$ \\
\hline Diclofenac sodium & $44(15 \%)$ & $24(12 \%)$ & $20(23 \%)$ & $1.85 \mathrm{E}-02 *$ \\
\hline Anticoagulant & $76(26 \%)$ & $35(17 \%)$ & $41(48 \%)$ & $4.46 \mathrm{E}-08^{* *}$ \\
\hline Heparin & $69(24 \%)$ & $32(15 \%)$ & $37(43 \%)$ & $1.54 \mathrm{E}-06^{* *}$ \\
\hline Antihypertensive agent & $67(23 \%)$ & $36(17 \%)$ & $31(36 \%)$ & $5.35 \mathrm{E}-04 * *$ \\
\hline
\end{tabular}




\begin{tabular}{|c|c|c|c|c|}
\hline & & & & (Continued) \\
\hline Medicine & Total $(n=293)$ & Moderate $(n=207)$ & Severe $(n=86)$ & $P$ values \\
\hline Amlodipine & $31(11 \%)$ & $14(7 \%)$ & $17(20 \%)$ & $2.77 \mathrm{E}-03 * *$ \\
\hline Immunomodulator & $62(21 \%)$ & $28(14 \%)$ & $34(40 \%)$ & $6.93 \mathrm{E}-07 * *$ \\
\hline Thymosin & $57(19 \%)$ & $24(12 \%)$ & $33(38 \%)$ & $5.18 \mathrm{E}-07^{* *}$ \\
\hline Antidiabetic & $51(17 \%)$ & $21(10 \%)$ & $30(35 \%)$ & $3.66 \mathrm{E}-07^{* *}$ \\
\hline Acarbose & $29(10 \%)$ & $15(7 \%)$ & $14(16 \%)$ & $2.97 \mathrm{E}-02 *$ \\
\hline Insulin & $28(10 \%)$ & $5(2 \%)$ & $23(27 \%)$ & $1.49 \mathrm{E}-09 * *$ \\
\hline Antishock & $22(8 \%)$ & $0(0 \%)$ & $22(26 \%)$ & $2.30 \mathrm{E}-13 * *$ \\
\hline Epinephrine & $19(6 \%)$ & $0(0 \%)$ & $19(22 \%)$ & $1.62 \mathrm{E}-11 * *$ \\
\hline Lobeline hydrochloride & $12(4 \%)$ & $0(0 \%)$ & $12(14 \%)$ & $2.30 \mathrm{E}-07^{* *}$ \\
\hline \multicolumn{5}{|l|}{ Prognosis } \\
\hline Discharged & $238(81 \%)$ & $198(96 \%)$ & $40(47 \%)$ & \\
\hline Transferred & $31(11 \%)$ & $9(4 \%)$ & $22(26 \%)$ & \\
\hline Died & $24(8 \%)$ & $0(0 \%)$ & $24(28 \%)$ & \\
\hline
\end{tabular}

Including drugs with statistical significance $(P$ values $<0.01)$ and top 20 drugs sorted by the number of patients in the total group. Data are presented as $n(\%)$ or $n / N(\%)$, where $N$ is the total number of patients in group. $P$ values were calculated via $\chi^{2}$ test or Fisher's exact test. $P$ values were used to compare the moderate and severe groups. ${ }^{*} P<0.05,{ }^{*} P<0.01$.

Table 6 Clinical therapies sorted by the prognosis of severe cases

\begin{tabular}{lllll}
\hline Medicine & Discharged $(n=40)$ & Transferred $(n=22)$ & Died $(n=24)$ & $P$ values \\
\hline Antibiotics & $38(95 \%)$ & $22(100 \%)$ & $23(96 \%)$ & $6.47 \mathrm{E}-01$ \\
Moxifloxacin & $37(93 \%)$ & $21(95 \%)$ & $22(92 \%)$ & $7.18 \mathrm{E}-01$ \\
Cefoperazone & $19(48 \%)$ & $15(68 \%)$ & $15(63 \%)$ & $2.44 \mathrm{E}-01$ \\
Imipenem and cilastatin sodium & $14(35 \%)$ & $13(59 \%)$ & $15(63 \%)$ & $3.24 \mathrm{E}-02 *$ \\
Antivira & $34(85 \%)$ & $21(95 \%)$ & $21(88 \%)$ & $9.26 \mathrm{E}-01$ \\
Arbidol & $24(60 \%)$ & $14(64 \%)$ & $18(75 \%)$ & $2.21 \mathrm{E}-01$ \\
Glucocorticoids & $30(75 \%)$ & $18(82 \%)$ & $19(79 \%)$ & $7.03 \mathrm{E}-01$ \\
Methylprednisolone & $28(70 \%)$ & $18(82 \%)$ & $19(79 \%)$ & $4.21 \mathrm{E}-01$ \\
TCM patent prescription & $29(73 \%)$ & $20(91 \%)$ & $23(96 \%)$ & $2.30 \mathrm{E}-02 *$ \\
Xue Bi Jing injection & $12(30 \%)$ & $7(32 \%)$ & $14(58 \%)$ & $2.55 \mathrm{E}-02 *$ \\
Antihypertensive agent & $15(38 \%)$ & $7(32 \%)$ & $9(38 \%)$ & $1.00 \mathrm{E}+00$ \\
Antishock & $0(0 \%)$ & $1(5 \%)$ & $21(86 \%)$ & $3.85 \mathrm{E}-12 * *$ \\
Epinephrine & $0(0 \%)$ & $0(0 \%)$ & $19(79 \%)$ & $4.87 \mathrm{E}-12 * *$ \\
\hline
\end{tabular}

Data are presented as $n(\%)$ or $n / N(\%)$, where $N$ is the total number of patients in group. $P$ values were calculated by using $\chi^{2}$ test or Fisher's exact test. $P$ values were used to compare the discharged and death groups. $* P<0.05, * * P<0.01$.

Table 7 Prognosis of patients treated with herbal prescriptions

\begin{tabular}{|c|c|c|c|}
\hline \multirow[t]{2}{*}{ Prognosis } & \multicolumn{3}{|c|}{$\begin{array}{c}\text { Disease conditions } \\
\text { (Number of patients treated with herb prescriptions/number of patients) }\end{array}$} \\
\hline & Moderate $(N=207)$ & Severe $(N=86)$ & Total $(N=293)$ \\
\hline Discharged $(n=238)$ & $187 / 198(94 \%)$ & $39 / 40(98 \%)$ & $226 / 238(95 \%)$ \\
\hline Transferred $(n=31)$ & 9/9 (100\%) & $21 / 22(95 \%)$ & $30 / 31(97 \%)$ \\
\hline $\operatorname{Died}(n=24)$ & $0 / 0$ & $17 / 24(71 \%)$ & $17 / 24(71 \%)$ \\
\hline Total $(n=293)$ & $196 / 207(95 \%)$ & $77 / 86(90 \%)$ & 273/293 (93\%) \\
\hline
\end{tabular}

In the TCM clinical setting of COVID-19, personalized herbal prescriptions remained regular therapeutic solutions for treatment. From our data, we found that 290 distinct herbs were used for COVID-19 treatment. Most of the herbs in these prescriptions had pharmacological effects (from TCM pharmacological perspectives) of clearing heat and detoxifying (e.g., Radix Scutellariae and Pericarpium Trichosanthis) and dampening phlegm (e.g., Rhizoma
Pinellia and Pericarpium Citri Reticulatae). These herbs had bitter or sweet properties and flavors. Despite the concept of holism that permeates all fields of TCM, including physiology, pathology, diagnosis, syndrome differentiation, and treatment, these therapies emphasized the upper energizer (heart and lung channel) and middle energizer (stomach and spleen channel) (Fig. 2B). Here, we showed and compared the partial results of herbal 

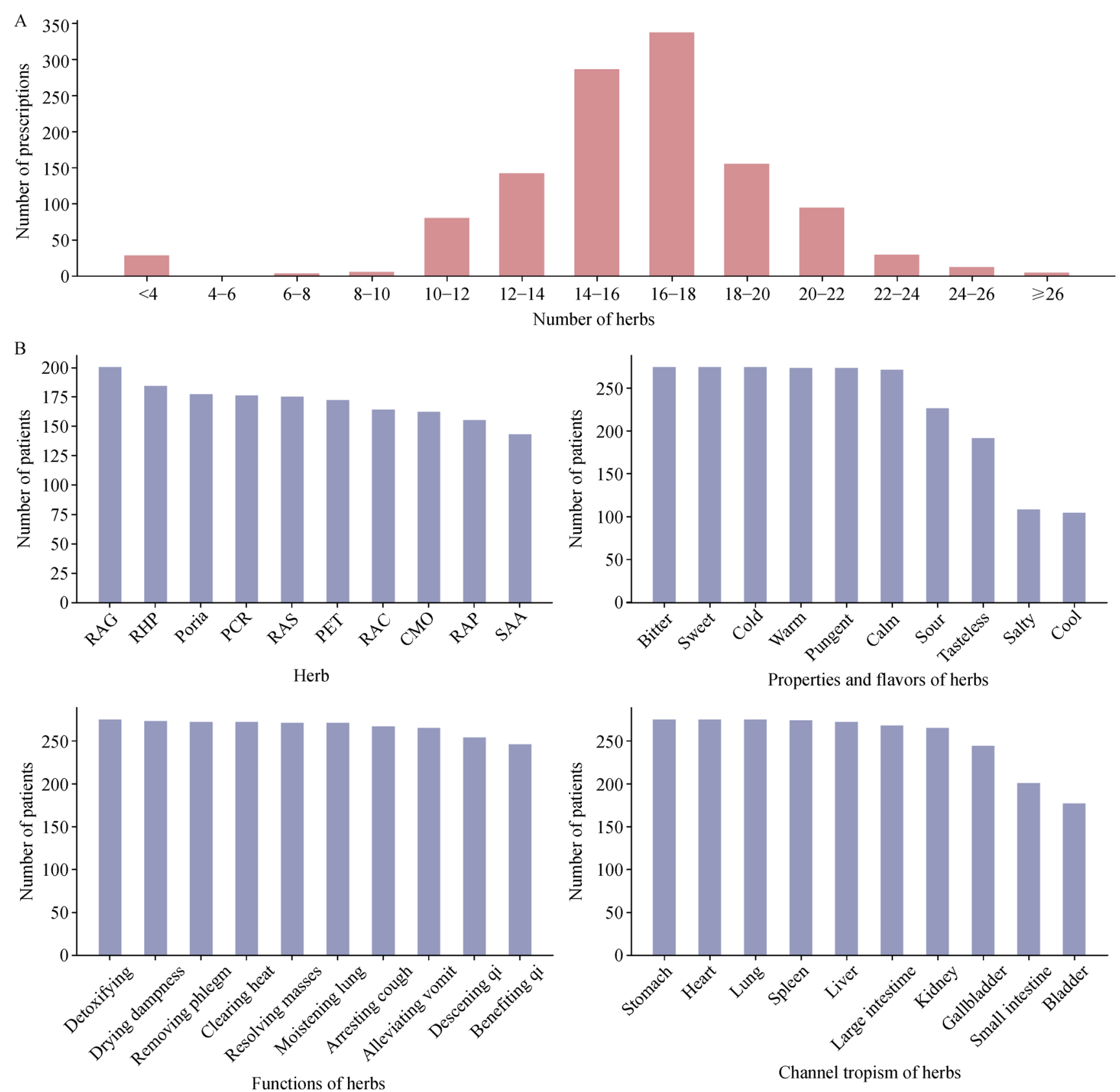

Fig. 2 Therapeutic characteristics of TCM herbal prescriptions. (A) Distributions of 1176 prescription-related herbs for 273 patients; (B) top 10 herbs and related features sorted by the number of patients. RAG, Radix Glycyrrhizae; RHP, Rhizoma Pinellia; PCR, Pericarpium Citri Reticulatae; RAS, Radix Scutellariae; PET, Pericarpium Trichosanthis; RAC, Radix Codonopsis; CMO, Cortex Magnoliae Officinalis; RAP, Radix Platycodonis; SAA, Semen Armeniacae Amarum.

therapies between moderate and severe cases (Tables 8 and 9).

Furthermore, we presented the top 10 herb combinations (Table 10) among the 1176 herb prescriptions to detect combination regularities (e.g., the frequently used herb combinations in TCM compound therapies) for the treatment of COVID-19 disease. The results showed that herb pairs, such as Semen Trichosanthis and Poria, Pericarpium Trichosanthis and Rhizoma Pinellia, and Pericarpium Citri Reticulatae and Radix Glycyrrhizae, were frequently prescribed for the treatment of patients with COVID-19.

We modeled herb combinations as a network and applied complex network analysis to identify the core herb combinations from these prescriptions (Fig. 3). The outcome was consistent with the results from association rule mining. The core combinations were confirmed to have the pharmacological effects of detoxification, phlegm removal, and dampness drying in COVID-19 treatment. 
Table 8 Differences between the herb therapies received by moderate and severe cases of COVID-19

\begin{tabular}{lllll}
\hline Herb & Total $(n=293)$ & Moderate $(n=207)$ & Severe $(n=86)$ & $P$ values \\
\hline Radix Glycyrrhizae & $200(68 \%)$ & $143(69 \%)$ & $57(66 \%)$ & $6.80 \mathrm{E}-01$ \\
Rhizoma Pinellia & $184(63 \%)$ & $137(66 \%)$ & $47(55 \%)$ & $8.41 \mathrm{E}-02$ \\
Poria & $177(60 \%)$ & $129(62 \%)$ & $48(56 \%)$ & $3.59 \mathrm{E}-01$ \\
Pericarpium Citri Reticulatae & $176(60 \%)$ & $130(63 \%)$ & $46(53 \%)$ & $1.51 \mathrm{E}-01$ \\
Radix Scutellariae & $175(60 \%)$ & $117(57 \%)$ & $58(67 \%)$ & $9.02 \mathrm{E}-02$ \\
Pericarpium Trichosanthis & $172(59 \%)$ & $124(60 \%)$ & $48(56 \%)$ & $5.18 \mathrm{E}-01$ \\
Radix Codonopsis & $164(56 \%)$ & $127(61 \%)$ & $37(43 \%)$ & $4.54 \mathrm{E}-03 * *$ \\
Cortex Magnoliae Officinalis & $162(55 \%)$ & $115(56 \%)$ & $47(55 \%)$ & $8.98 \mathrm{E}-01$ \\
Radix Platycodonis & $155(53 \%)$ & $121(58 \%)$ & $34(40 \%)$ & $4.54 \mathrm{E}-03 * *$ \\
Semen Armeniacae Amarum & $143(49 \%)$ & $100(48 \%)$ & $43(50 \%)$ & $7.99 \mathrm{E}-01$ \\
Rhizoma Anemarrhenae & $99(34 \%)$ & $62(30 \%)$ & $37(43 \%)$ & $4.15 \mathrm{E}-02 *$ \\
Fructus Schisandrae Chinensis & $75(26 \%)$ & $62(30 \%)$ & $13(15 \%)$ & $8.11 \mathrm{E}-03 * *$ \\
Fructus Forsythiae & $68(23 \%)$ & $41(20 \%)$ & $27(31 \%)$ & $4.75 \mathrm{E}-02^{*}$ \\
Radix Notoginseng & $55(19 \%)$ & $46(22 \%)$ & $9(10 \%)$ & $2.11 \mathrm{E}-02 *$ \\
Semen Coicis & $47(16 \%)$ & $27(13 \%)$ & $20(23 \%)$ & $3.62 \mathrm{E}-02 *$ \\
\hline
\end{tabular}

Including the top 10 herbs sorted on the basis of the number of patients and other five herbs with statistical significance. Data are presented as $n(\%)$ or $n / N(\%)$, where $N$ is the total number of patients in a group. $P$ values were calculated via $\chi^{2}$ test or Fisher's exact test and used to compare moderate and severe groups.

$* P<0.05, * * P<0.01$.

Table 9 Herb therapies sorted on the basis of the prognosis of patients with severe COVID-19

\begin{tabular}{lllll}
\hline Herb & Discharged $(n=40)$ & Transferred $(n=22)$ & Died $(n=24)$ & $P$ values \\
\hline Radix Scutellariae & $29(73 \%)$ & $16(73 \%)$ & $13(54 \%)$ & $1.77 \mathrm{E}-01$ \\
Radix Glycyrrhizae & $30(75 \%)$ & $15(68 \%)$ & $12(50 \%)$ & $5.81 \mathrm{E}-02$ \\
Pericarpium Trichosanthis & $29(73 \%)$ & $8(36 \%)$ & $11(46 \%)$ & $6.06 \mathrm{E}-02$ \\
Poria & $30(75 \%)$ & $10(45 \%)$ & $8(33 \%)$ & $1.54 \mathrm{E}-03 * *$ \\
Rhizoma Pinellia & $31(78 \%)$ & $6(27 \%)$ & $10(42 \%)$ & $6.61 \mathrm{E}-03 * *$ \\
Cortex Magnoliae Officinalis & $23(58 \%)$ & $11(50 \%)$ & $13(54 \%)$ & $8.01 \mathrm{E}-01$ \\
Pericarpium Citri Reticulatae & $24(60 \%)$ & $15(68 \%)$ & $7(29 \%)$ & $2.15 \mathrm{E}-02 *$ \\
Semen Armeniacae Amarum & $26(65 \%)$ & $10(45 \%)$ & $7(29 \%)$ & $9.26 \mathrm{E}-03 * *$ \\
Rhizoma Anemarrhenae & $21(53 \%)$ & $10(45 \%)$ & $6(25 \%)$ & $3.89 \mathrm{E}-02 *$ \\
Rhizoma Atractylodis Macrocephalae & $22(55 \%)$ & $10(45 \%)$ & $5(21 \%)$ & $9.37 \mathrm{E}-03 * *$ \\
Radix Codonopsis & $21(53 \%)$ & $10(45 \%)$ & $6(25 \%)$ & $3.89 \mathrm{E}-02 *$ \\
Radix Bupleuri & $19(48 \%)$ & $10(45 \%)$ & $7(29 \%)$ & $1.92 \mathrm{E}-01$ \\
Fructus Tsaoko & $18(45 \%)$ & $7(32 \%)$ & $10(42 \%)$ & $1.00 \mathrm{E}+00$ \\
Radix Ophiopogonis & $21(53 \%)$ & $10(45 \%)$ & $4(17 \%)$ & $7.45 \mathrm{E}-03 * *$ \\
Herba Pogostemonis & $18(45 \%)$ & $9(41 \%)$ & $7(29 \%)$ & $2.91 \mathrm{E}-01$ \\
\hline
\end{tabular}

Including the top 15 herbs sorted by the number of total patients. $P$ values were used to compare the discharged and death groups. Data are presented as $n(\%)$ or $n / N(\%)$, where $N$ is the total number of patients in each group. $P$ values were calculated by using $\chi^{2}$ test or Fisher's exact test. $P$ values were used to compare the discharged and death groups. $* P<0.05, * * P<0.01$.

Table 10 Top 10 herb combinations in COVID-19 TCM herbal prescriptions

\begin{tabular}{llll}
\hline Herb name & Herb name & Confidence & Frequency \\
\hline Poria & Semen Trichosanthis & 0.74 & 434 \\
Semen Trichosanthis & Poria & 0.95 & 434 \\
Pericarpium Trichosanthis & Rhizoma Pinellia & 0.78 & 398 \\
Pericarpium Citri Reticulatae & Radix Glycyrrhizae & 0.71 & 398 \\
Radix Codonopsis & Pericarpium Citri Reticulatae & 0.82 & 393 \\
Pericarpium Citri Reticulatae & Radix Codonopsis & 0.71 & 393 \\
Radix Scutellariae & Radix Glycyrrhizae & 0.79 & 388 \\
Radix Codonopsis & Radix Glycyrrhizae & 0.76 & 363 \\
Pericarpium Trichosanthis & Semen Trichosanthis & 0.70 & 358 \\
Semen Trichosanthis & Pericarpium Trichosanthis & 0.96 & 358 \\
\hline
\end{tabular}

Detected via association rules with support $>0.3$. The frequency indicates the number of herb pairs that co-occurred in prescriptions. 


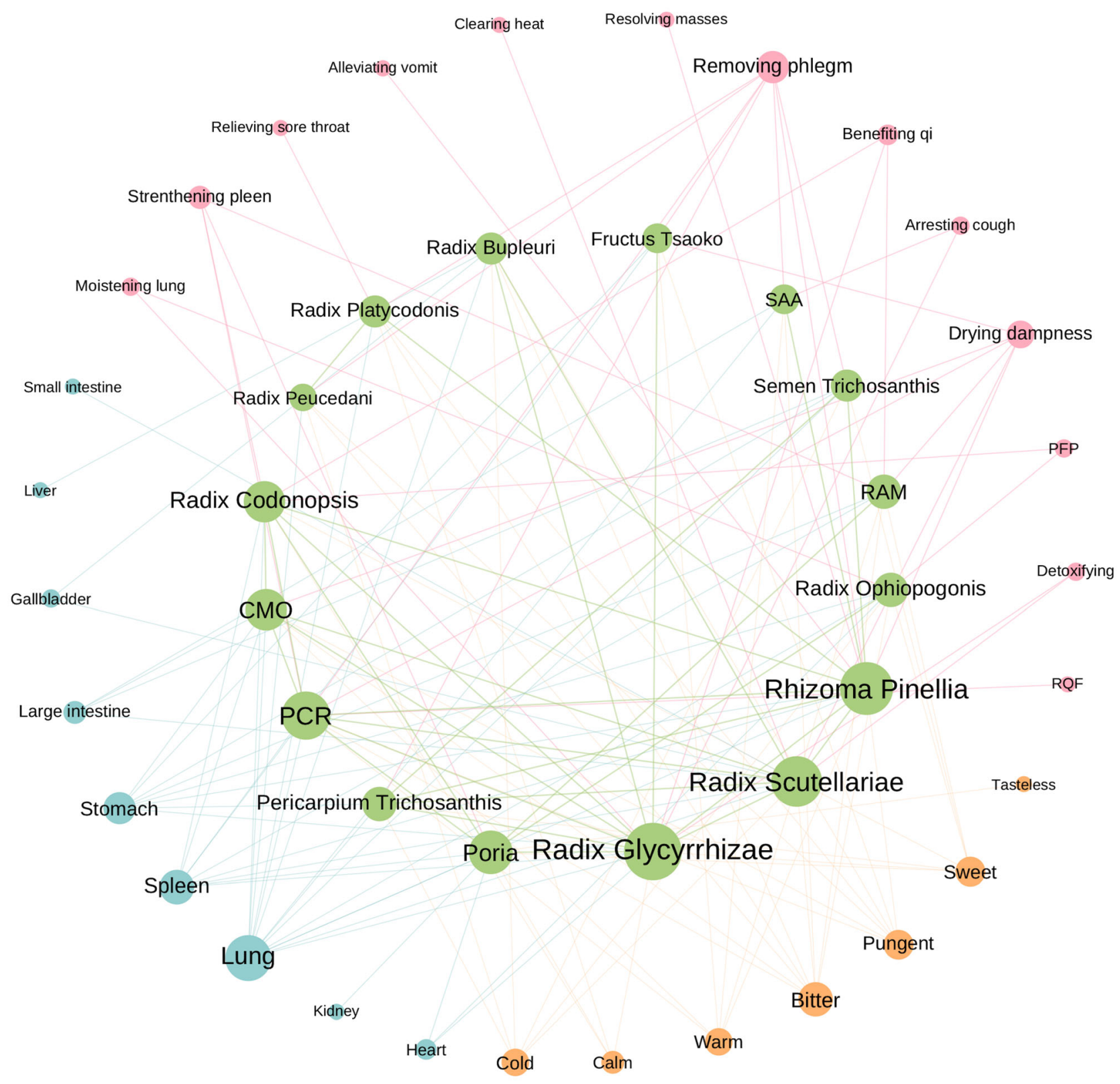

Fig. 3 Core herbs for COVID-19 treatment and their characteristics. This network shows the core herb combinations in TCM prescriptions. It is organized into four different types of nodes: the green nodes represent TCM herbs, and the pinkish blue and orange nodes correspond to the core pharmacological effects, channel tropisms, and flavor of the herbs. Node size reflects the degree value (a high degree is represented by a large node). PCR, Pericarpium Citri Reticulatae; CMO, Cortex Magnoliae Officinalis; SAA, Semen Armeniacae Amarum; RQF, Regulating qi flow; PFP, Rhizoma Atractylodis Macrocephalae; RAM, Rhizoma Atractylodis Macrocephalae.

\section{Therapeutic characteristics of therapies before admission}

We extracted the mentions of therapies in the text records of EMR admission notes to investigate the therapeutic profiles of patients with COVID-19 before admission. We identified 285 (97\%) patients with discomfort and found that $172(59 \%)$ patients were treated with drugs before admission (Table 11). Most of the patients received therapies with antibiotics (138) or antivirals (122). A total of $89(30 \%)$ patients were treated with TCM patent medicine. Among these patients, 69 (78\%) received Lianhuaqingwen capsule, which has a function of clearing heat and toxic materials. We observed that 83 symptoms (e.g., fever, cough, and fatigue) occurred before admission. 
Table 11 Therapeutic characteristics of preadmission therapies

\begin{tabular}{lll}
\hline Medicine & Patient number & Percentage $(n=293)$ \\
\hline Antibiotics & 138 & $47 \%$ \\
Moxifloxacin & 101 & $34 \%$ \\
Cephalosporin & 48 & $16 \%$ \\
Levofloxacin & 16 & $5 \%$ \\
Amoxicillin & 12 & $4 \%$ \\
Antivirals & 122 & $42 \%$ \\
Oseltamivir & 79 & $27 \%$ \\
Arbidol & 61 & $21 \%$ \\
Chinese patent medicine & 89 & $30 \%$ \\
Lianhuaqingwen capsule & 69 & $24 \%$ \\
Biological products & 14 & $5 \%$ \\
Immunoglobulin & 13 & $4 \%$ \\
\hline
\end{tabular}

Among the symptoms shown by the 94 pretreated patients, 51 had temporal outcomes (e.g., symptom disappearance) after preadmission treatment.

We found that the related symptoms relieved by the top 3 frequently used Western medical drugs before admission, namely, moxifloxacin (101 cases), oseltamivir (79 cases), and arbidol (61 cases), were fever (30\%-40\% partial relief rate (PRR)), fatigue and dry cough (20\%-45\% PRR), and cough (10\%-20\% PRR). However, these drugs rarely reduced the severity of chest tightness, palpitation, and wheezing. The related symptoms that could be relieved by the top Chinese patent medicine, Lianhuaqingwen capsule (69 cases), were fatigue (43\% PRR), fever (38\% PRR), dry cough (25\% PRR), coughing (20\% PRR), and wheezing (18\% PRR) (Table 12).

\section{Discussion}

Fully elucidating the patterns of the epidemiological and clinical features of COVID-19 is necessary to control, prevent, and treat this disease. TCM practitioners mainly investigate the patterns of symptom phenotypes for disease diagnosis and treatment. Therefore, obtaining the detailed TCM descriptions of patients with COVID-19 in terms of symptoms and tongue and pulse manifestations will help elucidate the clinical groups of patients with COVID-19. In addition, given the absence of proven evidence for the effectiveness of antiviral and antibiotic drugs [16-19], TCM herb prescriptions are a feasible clinical choice for COVID-19 treatment especially because they have been successfully used to treat SARS, a coronavirus-induced disease that is similar to COVID-19 [20-24]. In this retrospective study, we reported the clinical features of COVID-19 from the perspectives of TCM and Western medicine and further investigated related clinical therapies. In contrast to the cases that were included in previous studies [25-27], the 293 COVID-19 cases included in our analysis originated solely from a TCM hospital in Wuhan,
Table 12 Symptoms of preadmission medication cases with improvement

\begin{tabular}{|c|c|c|c|}
\hline $\begin{array}{l}\text { Medicine } \\
\text { and symptom }\end{array}$ & $\begin{array}{l}\text { Patient } \\
\text { number }\end{array}$ & $\begin{array}{l}\text { Patient } \\
\text { number } \\
\text { (relieved) }\end{array}$ & $\begin{array}{l}\text { Partial } \\
\text { relief rate }\end{array}$ \\
\hline \multicolumn{4}{|l|}{ Moxifloxacin } \\
\hline Fever & 63 & 27 & $43 \%$ \\
\hline Coughing & 36 & 7 & $19 \%$ \\
\hline Chest tightness & 25 & 1 & $4 \%$ \\
\hline Wheezing & 25 & 2 & $8 \%$ \\
\hline Dry cough & 10 & 2 & $20 \%$ \\
\hline Fatigue & 9 & 4 & $44 \%$ \\
\hline Diarrhea & 9 & 2 & $22 \%$ \\
\hline Palpitation & 4 & 0 & $0 \%$ \\
\hline Dry mouth & 4 & 0 & $0 \%$ \\
\hline Throat discomfort & 2 & 0 & $0 \%$ \\
\hline \multicolumn{4}{|l|}{ Oseltamivir } \\
\hline Fever & 44 & 13 & $30 \%$ \\
\hline Coughing & 29 & 3 & $10 \%$ \\
\hline Wheezing & 19 & 0 & $0 \%$ \\
\hline Chest tightness & 17 & 0 & $0 \%$ \\
\hline Diarrhea & 8 & 0 & $0 \%$ \\
\hline Dry cough & 7 & 0 & $0 \%$ \\
\hline Fatigue & 7 & 2 & $29 \%$ \\
\hline Palpitation & 5 & 0 & $0 \%$ \\
\hline Throat discomfort & 3 & 0 & $0 \%$ \\
\hline Dry mouth & 2 & 0 & $0 \%$ \\
\hline \multicolumn{4}{|l|}{ Arbidol } \\
\hline Fever & 30 & 12 & $40 \%$ \\
\hline Coughing & 21 & 4 & $19 \%$ \\
\hline Chest tightness & 17 & 3 & $18 \%$ \\
\hline Wheezing & 17 & 4 & $24 \%$ \\
\hline Diarrhea & 7 & 1 & $14 \%$ \\
\hline Dry cough & 7 & 3 & $43 \%$ \\
\hline Fatigue & 7 & 3 & $43 \%$ \\
\hline Palpitation & 3 & 0 & $0 \%$ \\
\hline Throat discomfort & 2 & 0 & $0 \%$ \\
\hline Dry mouth & 2 & 0 & $0 \%$ \\
\hline \multicolumn{4}{|c|}{ Lianhuaqingwen capsule } \\
\hline Fever & 39 & 15 & $39 \%$ \\
\hline Coughing & 25 & 5 & $20 \%$ \\
\hline Chest tightness & 18 & 2 & $11 \%$ \\
\hline Wheezing & 17 & 3 & $18 \%$ \\
\hline Dry cough & 12 & 3 & $25 \%$ \\
\hline Diarrhea & 7 & 0 & $0 \%$ \\
\hline Fatigue & 7 & 3 & $43 \%$ \\
\hline Palpitation & 4 & 0 & $0 \%$ \\
\hline Dry mouth & 2 & 0 & $0 \%$ \\
\hline Throat discomfort & 1 & 0 & $0 \%$ \\
\hline
\end{tabular}

where patients tended to be in severe condition and were elderly (57 years old on average). The long symptom onset time (10 days on average) before admission resulted in the 
overloading of hospitals in Wuhan during late January and the entirety of February. A total of $61 \%$ of the patients had different types of comorbidities, and the comorbidity rate of the 207 moderate cases approached 52\%. However, all the moderate cases were successfully treated, and most of these patients were discharged within 2 weeks. Among the remaining 86 severe cases, 40 were finally discharged successfully, and 24 died during treatment. The results showed that elderly male patients (73 years on average for death cases) with a high rate of disease comorbidity tended to have poor prognosis. The top frequently occurring symptoms were coughing, wheezing, chest tightness, fever, and insomnia. TCM-specific manifestations, such as yellow fur, might be a preventive TCM phenotype marker for good prognosis. Furthermore, most of the clinical findings from laboratory tests, such as white blood cell and lymphocyte counts, that were identified as risk factors for severe COVID-19 in our study were consistent with those reported in previous studies [28,29]. However, we found that triglycerides and total cholesterol were exceptions: the baseline levels of triglycerides and total cholesterol were higher in moderate cases than in severe cases but did not exceed their normal ranges. This result would be insightful for further biomedical investigations given that previous studies have shown that liver function and lipid levels are often related to viral infections in hepatitis B-related cirrhosis [30]. Cholesterol has critical functions in infection by several types of coronaviruses, such as type-I feline coronavirus [31,32].

Although regularly used drugs, such as methylprednisolone and moxifloxacin, have been widely applied to treat severe cases, reliable evidence for their effectiveness does not exist [16]. Therefore, screening potentially effective drugs for COVID-19 treatment is important. Among the 273 patients who used herbal prescriptions, 256 were discharged or transferred (i.e., $17 / 273=6.2 \%$ death rate for all cases and $17 / 77=22 \%$ death rate for severe cases). Among the remaining 20 patients who did not use herbal prescriptions, 13 survived $(7 / 20=35 \%$ death rate for all cases and $7 / 9=77.7 \%$ death rate for severe cases). These results hinted at the potential effectiveness of herbal prescriptions. Therefore, these prescriptions should be further studied in well-designed clinical trials. Our data covered a period of 1.5 months commencing from midJanuary, during which the national guideline for COVID19 treatment was still being developed. Frontline TCM practitioners developed optimal personalized prescriptions for patients with COVID-19. As a result, nearly 300 distinct herbs were used at that time. The results showed that common herbs and their combinations were practically prescribed for COVID-19 treatment. These common herbs and their related combinations, such as Poria, Pericarpium Trichosanthis and Radix Scutellariae, Pericarpium Tricho- santhis, and Radix Codonopsis and Fructus Forsythiae, have been included in the suggested prescriptions in the latest national guideline of COVID-19 [33]. The pharmacological effects of these herbs in removing phlegm, clearing heat, and arresting cough were useful in combating cough, fever, phlegm, and COVID-19-related complications. Moreover, the herbs, such as Radix Ophiopogonis, Poria, Rhizoma Pinellia, and Rhizoma Atractylodis Macrocephalae, possibly contributed to the good prognosis of severe cases. The underlying pharmacological mechanisms of these herbs and their related prescriptions for COVID-19 treatment have been active research topics in recent months [34]. We found that the three popularly used drugs, namely moxifloxacin, oseltamivir, and abidol, and one Chinese patent medicine (i.e., Lianhuaqingwen capsule) provided some degree of symptom (e.g., fever and dry cough) relief. However, these drugs rarely relieved most symptoms, such as chest tightness, palpitation, and wheezing, related to severe conditions. Western drugs were given to patients who stayed in-hospital, and 238 (81.2\%) cases were finally discharged. The results indicated the effectiveness of TCM herbal prescriptions in the integrative treatment of COVID-19.

Our study has several limitations. First, given that the causative pathogen had just been identified at the time of admission, the diagnoses of most of the 293 patients were confirmed to be positive on the basis of the presence of SARS-CoV-2 nucleic acid. However, 102 cases still lacked viral RNA detective data. Second, this study involved a modest set of cases of hospitalized patients. Therefore, the laboratory test results, especially the multiple test comparisons, statistical test results, and $P$ values obtained in this work, should be interpreted with caution because of the limited number of cases. Third, 31 patients were transferred to designated hospitals for mixed reasons, including management reasons in the early days of the pandemic and condition aggravation. Therefore, assessing the prognoses of these cases is difficult. Finally, this study mainly focused on the clinical features of the real-world manifestations and therapeutic characteristics of the integrated treatment of 293 COVID-19 cases in a clinical environment in Wuhan, China. Carefully designed clinical trials are required to filter out potential confounders to validate the correlations between the clinical features and prognosis of COVID-19 that may have clinical implications. Furthermore, due to complicated interactions among clinical features (e.g., symptoms and comorbidities) $[35,36]$ and associations between phenotypes and genotypes, the exploration of the correlations between clinical phenotype networks and prognosis by COVID-19 clinical management is promising [37-39] to identify the precise patient subgroups $[40,41]$ with possibly high mortality or good prognosis. 


\section{Conclusions}

We described the clinical features and therapeutic characteristics of 293 COVID-19 inpatient cases in Wuhan. These cases were treated via the integration of TCM herbal prescriptions and Western medicine. Our results reconfirmed several well-recognized risk factors, such as age, gender (male), and comorbidities, and provided novel laboratory indications (e.g., cholesterol) and TCM-specific phenotype markers (e.g., dull tongue) that were relevant to COVID-19 infections and prognosis. Furthermore, we proposed a TCM symptom profile on the basis of nearly 300 COVID-19 cases. This profile would provide some important insights into TCM pathological studies. We identified frequently used herbs and their combinations from 1176 TCM prescriptions, which reflected the treatment principles of TCM for COVID-19 infections. Lastly, we found that $59 \%$ of patients received some form of treatment, including antivirals, antibiotics, and Chinese patent medicine, before admission. These might have some effects on COVID-19 symptoms, such as fever and dry cough.

\section{Acknowledgements}

This work is partially supported by the National Key Research and Development Program (Nos. 2017YFC1703506, 2017YFC1703505, 2017YFC1703502, and 2020YFC0841600), the Special Programs of Traditional Chinese Medicine (Nos. JDZX2015168, JDZX2015171, and JDZX2015170) and the Fundamental Research Funds for the Central public welfare research institutes (Nos. ZZ10-005 and 2018JBZ006).

\section{Compliance with ethics guidelines}

Zixin Shu, Yana Zhou, Kai Chang, Jifen Liu, Xiaojun Min, Qing Zhang, Jing Sun, Yajuan Xiong, Qunsheng Zou, Qiguang Zheng, Jinghui Ji, Josiah Poon, Baoyan Liu, Xuezhong Zhou, and Xiaodong Li declare that they have no conflict of interest. This study was approved by the ethics review board of Hubei Provincial Hospital of Traditional Chinese Medicine (HBZY2020-C01-01). Written consent was waived due to the retrospective nature. This study was reported according to STROBE (The Strengthening the Reporting of Observational Studies in Epidemiology).

\section{References}

1. Jiang F, Deng L, Zhang L, Cai Y, Cheung CW, Xia Z. Review of the clinical characteristics of coronavirus disease 2019 (COVID-19). J Gen Intern Med 2020; 35(5): 1545-1549

2. Huang C, Wang Y, Li X, Ren L, Zhao J, Hu Y, Zhang L, Fan G, Xu J, Gu X, Cheng Z, Yu T, Xia J, Wei Y, Wu W, Xie X, Yin W, Li H, Liu M, Xiao Y, Gao H, Guo L, Xie J, Wang G, Jiang R, Gao Z, Jin Q, Wang J, Cao B. Clinical features of patients infected with 2019 novel coronavirus in Wuhan, China. Lancet 2020; 395 (10223): 497-506

3. Chan JF, Yuan S, Kok KH, To KK, Chu H, Yang J, Xing F, Liu J, Yip CC, Poon RW, Tsoi HW, Lo SK, Chan KH, Poon VK, Chan WM, Ip JD, Cai JP, Cheng VC, Chen H, Hui CK, Yuen KY. A familial cluster of pneumonia associated with the 2019 novel coronavirus indicating person-to-person transmission: a study of a family cluster. Lancet 2020; 395(10223): 514-523

4. The Lancet. Emerging understandings of 2019-nCoV. Lancet 2020; 395(10221): 311

5. Chan KW, Wong VT, Tang SCW. COVID-19: an update on the epidemiological, clinical, preventive and therapeutic evidence and guidelines of integrative Chinese-Western medicine for the management of 2019 novel coronavirus disease. Am J Chin Med 2020; 48(3): 737-762

6. Lu YF, Yang ZG, Wang M, Shi J, Wang ZW, LYU Y, Tang BZ, Ye $\mathrm{C}, \mathrm{Xu}$ QN, Yin KS, Chen XR. Analysis on Chinese medical clinical characteristics of 50 patients with 2019-nCoV-infected pneumonia. J Shanghai Univ Tradit Chin Med (Shanghai Zhong Yi Yao Da Xue Xue Bao) 2020; (2): 17-21 (in Chinese)

7. Zheng WK, Zhang JH, Yang FW, Wang YG, Liu QQ, Zhang BL. Comprehensive analysis of diagnosis and treatment schemes for prevention and treatment of novel coronavirus pneumonia by traditional Chinese medicine. J Tradit Chin Med (Zhong Yi Za Zhi) 2020; 61(4): 277-280 (in Chinese)

8. Jin YH, Cai L, Cheng ZS, Cheng H, Deng T, Fan YP, Fang C, Huang D, Huang LQ, Huang Q, Han Y, Hu B, Hu F, Li BH, Li YR, Liang K, Lin LK, Luo LS, Ma J, Ma LL, Peng ZY, Pan YB, Pan ZY, Ren XQ, Sun HM, Wang Y, Wang YY, Weng H, Wei CJ, Wu DF, Xia J, Xiong Y, Xu HB, Yao XM, Yuan YF, Ye TS, Zhang XC, Zhang YW, Zhang YG, Zhang HM, Zhao Y, Zhao MJ, Zi H, Zeng XT, Wang YY, Wang XH; the Zhongnan Hospital of Wuhan University Novel Coronavirus Management and Research Team, Evidence-Based Medicine Chapter of China International Exchange and Promotive Association for Medical and Health Care (CPAM). A rapid advice guideline for the diagnosis and treatment of 2019 novel coronavirus (2019-nCoV) infected pneumonia (standard version). Mil Med Res 2020; 7(1): 4

9. Liang TB. Handbook of COVID-19 prevention and treatment. The First Affiliated Hospital, Zhejiang University School of Medicine. Compiled According to Clinical Experience. 2020. http://che.zju. edu.cn/cheen/2020/0401/c27758a2021088/page.htm (accessed March 20, 2020)

10. Wang Y, Wang L, Rastegar-Mojarad M, Moon S, Shen F, Afzal N, Liu S, Zeng Y, Mehrabi S, Sohn S, Liu H. Clinical information extraction applications: a literature review. J Biomed Inform 2018; 77:34-49

11. Reed GM. Toward ICD-11: improving the clinical utility of WHO's International Classification of mental disorders. Prof Psychol Res Pr 2010; 41(6): 457-464

12. Baclawski K, Matheus CJ, Kokar MM, Letkowski J,Kogut PA. Towards a symptom ontology for semantic web applications. In: Proceedings of Third International Semantic Web Conference. Hiroshima, Japan. 2004. 650-667

13. Zhang Y, Xie YM, Li YN, Zhang C, Chen C, Zhuang Y. Association rules analysis for exploring combined medication characteristics of Fufang Kushen injection: real-world study based on 49597 cases. China J Chin Mater Medica (Zhongguo Zhong Yao 
Za Zhi) 2017; 42(15): 2900-2904 (in Chinese)

14. Müller B, Reinhardt J, Strickland MT. Neural Networks: An Introduction. Springer, Berlin/Heidelberg, Germany. 2012

15. Yang Y, Yang K, Hao T, Zhu G, Ling R, Zhou X, Li P. Prediction of molecular mechanisms for Lianxia Ningxin Formula: a network pharmacology study. Front Physiol 2018; 9:489

16. FitzGerald GA. Misguided drug advice for COVID-19. Science 2020; 367(6485): 1434

17. Russell B, Moss C, George G, Santaolalla A, Cope A, Papa S, Van Hemelrijck M. Associations between immune-suppressive and stimulating drugs and novel COVID-19-a systematic review of current evidence. Ecancermedicalscience 2020; 14:1022

18. Md Insiat Islam Rabby. Current drugs with potential for treatment of COVID-19: a literature review. J Pharm Pharm Sci 2020; 23(1): 58 64

19. Zhang W, Zhao Y, Zhang F, Wang Q, Li T, Liu Z, Wang J, Qin Y, Zhang X, Yan X, Zeng X, Zhang S. The use of anti-inflammatory drugs in the treatment of people with severe coronavirus disease 2019 (COVID-19): the perspectives of clinical immunologists from China. Clin Immunol 2020; 214: 108393

20. Zeng SP. Study of integrated traditional Chinese and western medicine treatment for severe COVID-19. Classical Chin Med Res (Jing Dian Zhong Yi Yan Jiu) 2020; 3(2): 36-45 (in Chinese)

21. He LL, Gong PY, Feng Y, Zou W,Wang EL, Gu J. Analysis on application of Chinese materia medica in treatment of COVID-19 by suppressing cytokine storm. Chin Tradit Herbal Drugs (Zhong Cao Yao) 2020; 51(6): 1375-1385 (in Chinese)

22. Ling CQ. Traditional Chinese medicine is a resource for drug discovery against 2019 novel coronavirus (SARS-CoV-2). J Integr Med 2020; 18(2): 87-88

23. Li SY, Chen C, Zhang HQ, Guo HY, Wang H, Wang L, Zhang X, Hua SN, Yu J, Xiao PG, Li RS, Tan X. Identification of natural compounds with antiviral activities against SARS-associated coronavirus. Antiviral Res 2005; 67(1): 18-23

24. Ran J, Li YP, Li QT, Liu HB, Zeng CF, Ren Y, Wan P, Wei DR, Li XH, Yang MG, Wu MS, Zuo GQ, He LF, Wang HW, Li PF, Luo L. Study of TCM syndrome in 209 novel coronavirus pneumonia cases of Chongqing in 2020. J Emerg Tradit Chin Med (Zhongguo Zhong Yi Ji Zheng) 2020; 29(5): 753-755, 758 (in Chinese)

25. Yang JY, Sun W, Qiao J, Cai R, Liu X, Wei L. Analysis on traditional Chinese medicine syndromes and constitutions of 90 patients with common COVID-19. J Tradit Chin Med (Zhong Yi Za Zhi) 2020; 61(8): 645-649 (in Chinese)

26. Sun HY, Bi YF, Zhu ZG, Liu M, Liu XQ, Ma ZR, Wang XL, Zhao GY, Su Q, Mao JY, Zhang BL. Characteristics of traditional Chinese medicine syndromes in 88 patients with coronavirus diseases 2019 in Tianjin. J Tradit Chin Med (Zhong Yi Za Zhi) 2020; 61(10): 837-841 (in Chinese)

27. Li JS, Li SY, Xie X, Zhao HL, Feng ZZ, Chun L, Wang JJ, Sun BQ. Clinical characteristics and TCM syndrome distribution of 524 COVID-19 patients in Henan province. J Tradit Chin Med (Zhong Yi Za Zhi) 2020; [Epub ahead of print] (in Chinese) http://kns.cnki. net/kcms/detail/11.2166.R.20200311.1508.002.html
28. Wang D, Hu B, Hu C, Zhu F, Liu X, Zhang J, Wang B, Xiang H, Cheng Z, Xiong Y, Zhao Y, Li Y, Wang X, Peng Z. Clinical characteristics of 138 hospitalized patients with 2019 novel coronavirus-infected pneumonia in Wuhan, China. JAMA 2020; 323(11): 1061-1069

29. Yang X, Yu Y, Xu J, Shu H, Xia J, Liu H, Wu Y, Zhang L, Yu Z, Fang M, Yu T, Wang Y, Pan S, Zou X, Yuan S, Shang Y. Clinical course and outcomes of critically ill patients with SARS-CoV-2 pneumonia in Wuhan, China: a single-centered, retrospective, observational study. Lancet Respir Med 2020; 8(5): 475-481

30. Cao WJ, Wang TT, Gao YF, Wang YQ, Bao T, Zou GZ. Serum lipid metabolic derangement is associated with disease progression during chronic HBV infection. Clin Lab 2019; 65(12): 23772386

31. Schroeder C. Cholesterol-binding viral proteins in virus entry and morphogenesis. Subcell Biochem 2010; 51:77-108

32. Takano T, Endoh M, Fukatsu H, Sakurada H, Doki T, Hohdatsu T. The cholesterol transport inhibitor U18666A inhibits type I feline coronavirus infection. Antiviral Res 2017; 145: 96-102

33. National Health Commission. The Diagnosis and Treatment Protocol for Novel Coronavirus Pneumonia (Trial Version Seven). 2020. http://www.nhc.gov.cn/yzygj/s7653p/202003/ 46c9294a7dfe4cef80dc7f5912eb1989.shtml (in Chinese) (accessed March 9, 2020)

34. Zhang DH, Wu KL, Zhang X, Deng SQ, Peng B. In silico screening of Chinese herbal medicines with the potential to directly inhibit 2019 novel coronavirus. J Integr Med 2020; 18(2): 152-158

35. Chakrabarty B, Das D, Bulusu G. Network-based analysis of fatal comorbidities of COVID-19 and potential therapeutics. ChemRxiv 2020; doi: 10.26434/chemrxiv.12136470.v1

36. Maddaloni E, Buzzetti R. COVID-19 and diabetes mellitus: unveiling the interaction of two pandemics. Diabetes Metab Res Rev 2020; [Epub ahead of print] doi: 10.1002/dmrr.3321

37. Zhou X, Li Y, Peng Y, Hu J, Zhang R, He L, Wang Y, Jiang L, Yan S, Li P, Xie Q, Liu B. Clinical phenotype network: the underlying mechanism for personalized diagnosis and treatment of traditional Chinese medicine. Front Med 2014; 8(3): 337-346

38. Huang M, Wang J, Zhang R, Ni Z, Liu X, Liu W, Kong W, Chen Y, Huang T, Li G, Wei D, Liu J, Zhou X. Symptom network topological features predict the effectiveness of herbal treatment for pediatric cough. Front Med 2020; 14(3): 357-367

39. Barabási AL, Gulbahce N, Loscalzo J. Network medicine: a network-based approach to human disease. Nat Rev Genet 2011; 12 (1): $56-68$

40. Zhou X, Lei L, Liu J, Halu A, Zhang Y, Li B, Guo Z, Liu G, Sun C, Loscalzo J, Sharma A, Wang Z. A systems approach to refine disease taxonomy by integrating phenotypic and molecular networks. EBioMedicine 2018; 31: 79-91

41. Shu Z, Liu W, Wu H, Xiao M, Wu D, Cao T, Ren M, Tao J, Zhang C, He T, Li X, Zhang R, Zhou X. Symptom-based network classification identifies distinct clinical subgroups of liver diseases with common molecular pathways. Comput Methods Programs Biomed 2019; 174: 41-50 